\title{
Functional Ovarian Cyst
}

National Cancer Institute

\section{Source}

National Cancer Institute. Functional Ovarian Cyst. NCI Thesaurus. Code C5216.

A benign ovarian cyst that develops and regresses during the menstrual cycle as part of normal ovarian function. 